\title{
The highlights of the Pierre Auger Observatory
}

\author{
Lorenzo Caccianiga $^{* a}$ for the Pierre Auger Collaboration ${ }^{b}$ \\ ${ }^{a}$ Istituto Nazionale di Fisica Nucleare - Sezione di Milano, Italy \\ Full author list: http://www.auger.org/archive/authors_2019_12.html \\ ${ }^{b}$ Av. San Martín Norte 304 (5613) Malargüe, Prov. de Mendoza, Argentina \\ E-mail: lorenzo.caccianiga@mi.infn.it
}

\begin{abstract}
The Pierre Auger Observatory, in Argentina, is the largest cosmic ray detector ever built. It has actively been taking data for more than 15 years now, and thanks to this detector, we have a better understanding of the most energetic particles in the universe. We can now start to answer some of the most important questions, such as where do they come from? What are they? How do they interact with the atmosphere? In this proceedings, a selection of the most important results of Auger from the past few years is presented, with particular focus on those of astrophysical interest.
\end{abstract}

Multifrequency Behaviour of High Energy Cosmic Sources - XIII - MULTIF2019

3-8 June 2019

Palermo, Italy

${ }^{*}$ Speaker. 


\section{The Pierre Auger Observatory}

The Pierre Auger Observatory [1] is the largest detector of ultra-high energy cosmic rays (UHECR) ever built, covering $\sim 3000 \mathrm{~km}^{2}$ of pampa amarilla in Argentina near the town of Malarguie. It can observe extensive air showers (EAS) induced by the interaction of UHECRs with the atmosphere with independent and complementary instruments (hybrid design): the main two are the fluorescence detector (FD), and the surface detector array (SD). The Observatory has been taking data since 2004 and was completed in 2008. The SD is composed of 1660 water-Cherenkov detectors (WCDs) on a triangular grid of $1500 \mathrm{~m}$ spacing (standard array) that ensures a full detection efficiency above $3 \mathrm{EeV}^{1}$. A denser spacing ( $750 \mathrm{~m}$, infill array) has been later implemented in a small area of the array, thus lowering the energy threshold of full detection efficiency down to $0.3 \mathrm{EeV}$. The FD consists of 24 telescopes in four sites that overlook the atmosphere above the SD. An extension to cover lower energies has been deployed for FD too: three telescopes have been added in the infill region with a higher elevation to look at the lower energy showers, which develop higher in the atmosphere. The FD measures the fluorescence light emitted by the molecules excited by the shower as it propagates in the atmosphere, which is proportional to the energy deposited by shower particles. Hence, the FD is capable of performing a quasi-calorimetric measurement of the primary cosmic ray energy. However, since it can operate only on clear moonless nights, its duty cycle is limited to $\sim 15 \%$. On the other hand, the SD has a $\sim 100 \%$ duty cycle but can only measure the lateral development of the shower, and cannot access the primary energy directly. The advantage of a hybrid detector is that, through the events observed by both detectors (i.e. hybrid events), it is possible to perform a calibration of the SD and compute the energy of SD-only events without the need of Monte Carlo simulations. The energy resolution after this process is of the order of $\sim 15 \%$. Additional detectors are installed at the observatory site, in particular, underground muon detectors and the AERA array [27] that measures the radio signals emitted by the EAS. An important upgrade of the Observatory is being deployed at the time of writing and will be described in detail in section 6.

\section{Energy Spectrum}

One of the main goals of the Pierre Auger Observatory is to measure the cosmic ray spectrum at its highest energy end with unprecedented precision. We use data collected by the different detectors of the Observatory, divided into different samples. The SD-1500m produces two different samples, the first contains "vertical" events (zenith angle $\theta<60^{\circ}$ ) and the second contains "horizontal" events $\left(60^{\circ}<\theta<80^{\circ}\right)$; these samples have different reconstruction techniques and thus are kept separated in the analysis. An additional sample comes from the infill SD array, that uses only vertical events $\left(\theta<55^{\circ}\right)$. The FD produces two samples: the main one composed of hybrid data and, since recently, also a sample of low energy events (down to less than $10^{17} \mathrm{eV}$ for which the FD has observed the Cherenkov emission from their shower, rather than the fluorescence one [6]. All the spectra are then combined through a maximum likelihood fit to obtain the final spectrum [3]. The data show with high significance two inflection points usually referred to as the secondknee [7] and ankle in addition to the clear flux suppression that is observed at energies higher than

\footnotetext{
${ }^{1} 1 \mathrm{EeV}=10^{18} \mathrm{eV}$
} 
$\sim 5 \times 10^{19} \mathrm{eV}$. In addition to those, an indication of a new spectral feature around $\sim 10^{19} \mathrm{eV}$ has emerged with the unprecedented statistics of the latest analysis.

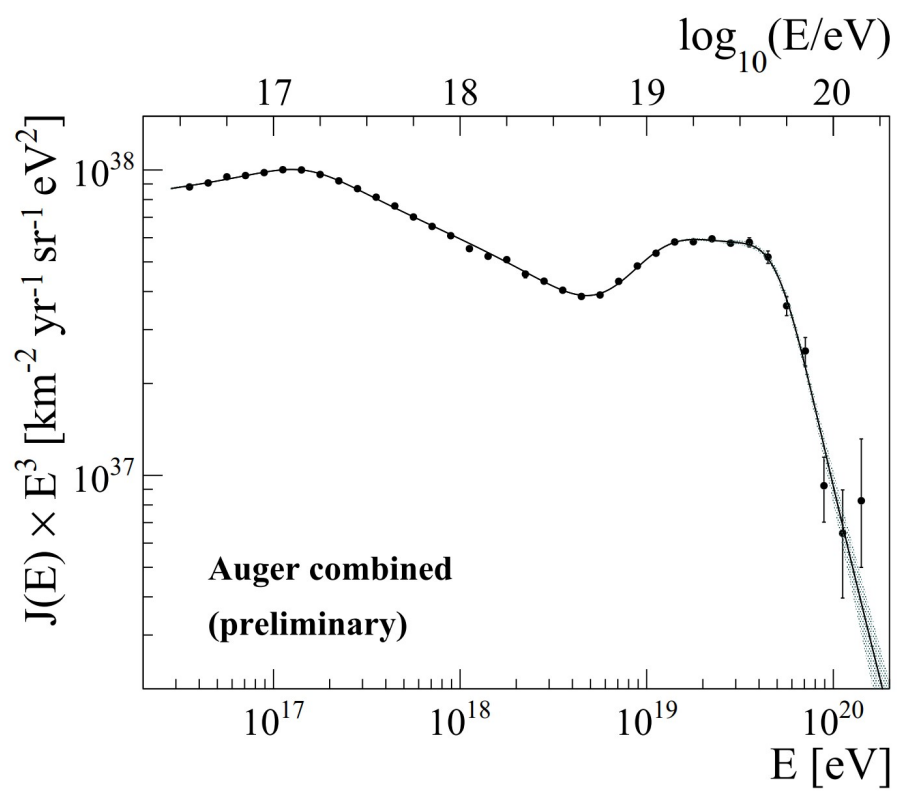

Figure 1: The most up-to date combined spectrum of UHECRs as measured by the Auger Observatory. [3]

\section{Composition}

The composition of the UHECR flux is an important parameter in the quest for its sources. The best mass-related observable accessible to Auger is $X_{\max }$, i.e. the depth of the maximum of the particle production in the shower. Direct measurements of $X_{\max }$ can be done only through the FD, and thus only on a fraction of Auger events. The most recent results [8] are shown in figure 2 where the first and second moments, $X_{\max }$ and $\sigma\left(X_{\max }\right)$ of the measured $X_{\max }$ distribution are plotted as a function of energy.

Information on the mass can be obtained by comparing the measurements with the prediction from simulations, which however, are made based on hadronic interaction models extrapolated from lower energies (the ones accessible with accelerators). Since different models can have very different predictions, it is not possible to extract precise estimates of the composition at each energy. However, some important results can still be achieved in a model-independent scenario: first of all, we can affirm that the composition gets lighter up to $2 \mathrm{EeV}$ and heavier afterwards. Moreover, in the $2-5 \mathrm{EeV}$ range, a pure composition is excluded together with proton-helium mixes [11]. Analyses based on the SD only, with its higher statistics, are also performed [12, 25], and confirm the findings previously reported. Moreover, they also show that simulations made with the currently available hadronic interaction models do not correctly reproduce the physics of extensive air showers. 

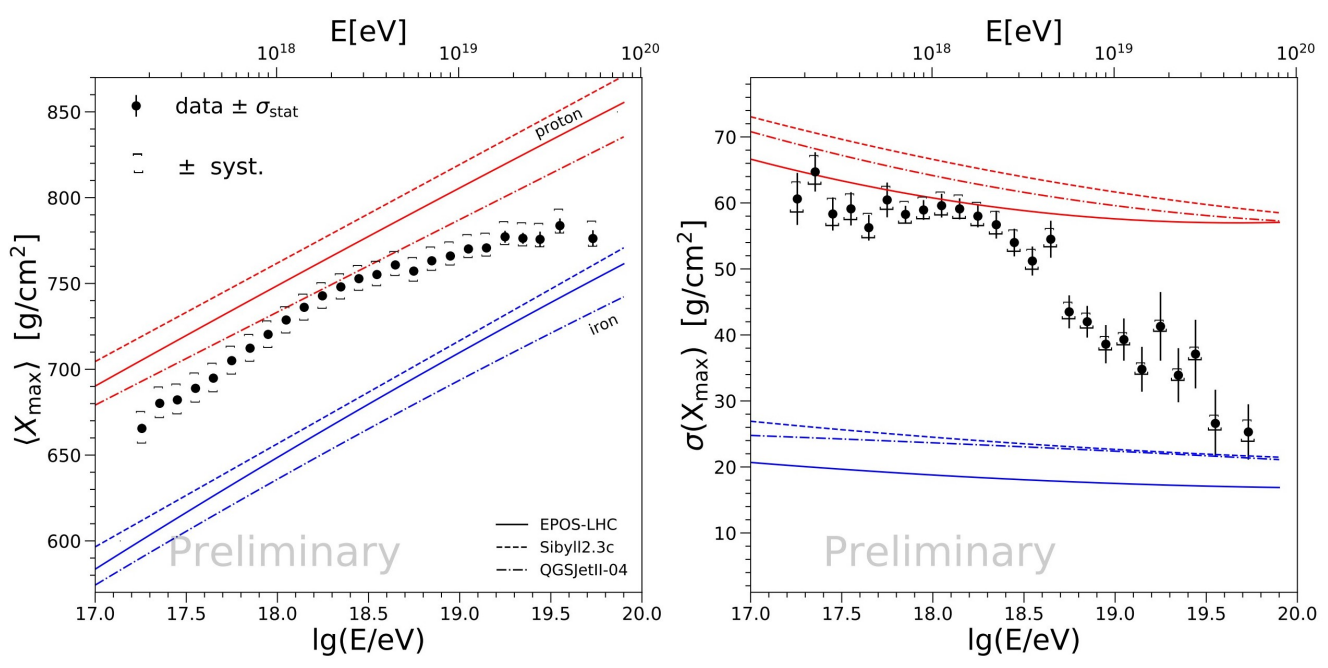

Figure 2: Mean $X_{\max }($ left $)$ and $\sigma\left(X_{\max }\right)$ (right) as a function of energy compared to expectations from simulations for proton and iron primaries. From [8]

\section{Photons and neutrinos}

Auger is also capable of observing showers generated by ultra-high energy photons and neutrinos. Distinguishing them from EAS initiated by cosmic rays is not trivial and is done with different methods involving both SD and FD. For both neutrinos and photons, no unambiguous detection has been yet achieved $[9,10]$, and upper limits have been set. Regarding photons, these upper limits severely constrain "exotic" models for the origin of UHECRs (so-called "top-down") and are now close to reaching the flux expected from GZK photons, i.e. photons produced after a proton CR has interacted with the photon background producing a pion and losing energy. Current best limits from Auger compared to predictions and other experiments are shown in figure 3.

We searched for neutrinos in coincidence with transient events such as gravitational wave events and the bursts from TXS 0506+056, a blazar that was observed to emit neutrinos correlated to the photon luminosity, although at lower energies than the ones accessible by the Pierre Auger Observatory. No candidate neutrino was found for either search, and upper limits were set. In particular, given the number of BBH mergers observed by the LIGO-Virgo collaboration, we combined the follow-up for all those events to set an upper limit on a universal isotropic UHE neutrino luminosity as a function of time after the merger. Since no UHE neutrino was observed in the merger localization regions in a 24-hour period after each event, an upper limit on the luminosity $L_{\text {up }} \sim 4.3 \times 10^{46} \mathrm{erg} / \mathrm{s}$ is set, under the assumption that the sources emit UHE neutrinos with a spectrum $\propto E_{v}^{-2}$.

\section{Arrival directions}

The study of the arrival directions of UHECRs is probably the most natural way to search for their sources. Unfortunately, as cosmic rays are charged particles, this is made difficult because of the deflections they suffer when passing through the Galactic and extragalactic magnetic fields. 


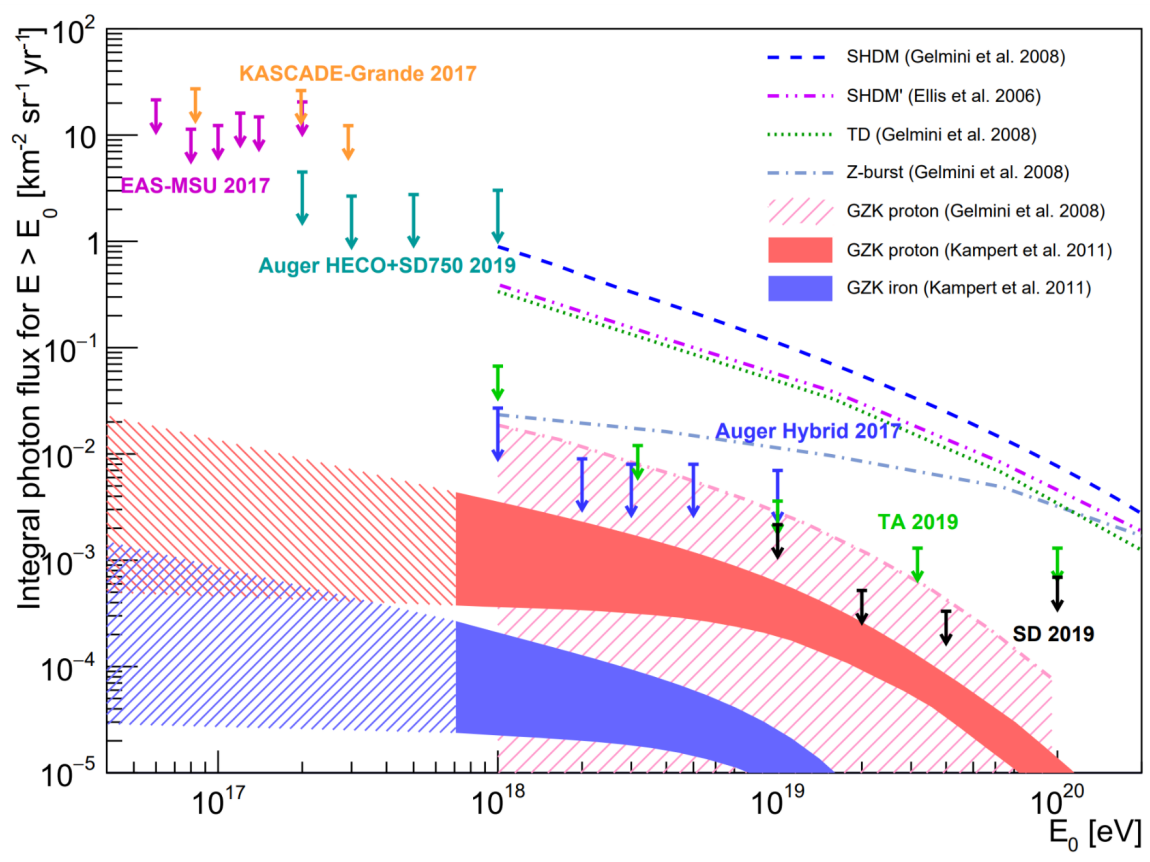

Figure 3: Photon flux limits at 95\% C.L. for different analyses by the Pierre Auger Observatory, compared to other measurements and predictions from different models. For further details, see [9].

The precise intensity and direction of such fields are unknown, but we expect deflections of the order of a few degrees for a $100 \mathrm{EeV}$ proton in most parts of the GMF. The search for sources can then be done by either looking at the highest energies, where deflections should be the least, and searching for small and intermediate scale anisotropies, or looking at lower energies for large scale anisotropies. The latter search can still give useful information on the distribution of the sources even in the case of strong magnetic fields because anisotropy cannot arise through deflections of an originally isotropic flux by a magnetic field, following Liouville's theorem. The Pierre Auger Observatory observed a large scale anisotropy [2] for events with energy higher than $8 \mathrm{EeV}$. In this energy range, the amplitude of the first harmonic was found to be incompatible with an isotropic distribution at a level of more than $5.2 \sigma$. In this energy bin, an extragalactic origin of UHECR is favored since the dipole direction is not compatible with what would be expected from a Galactic origin (the dipole direction points $\sim 125^{\circ}$ away from the Galactic center), even taking into account magnetic deflections. On the other hand, the same analysis on events in the 4-8 EeV energy range leads to a result compatible with isotropy. If the sources of UHECRs in this energy bin were Galactic, we would expect to see anisotropy, and only in the case of a heavy composition, one could expect this anisotropy to be suppressed by magnetic fields to the point of being undetectable. Since composition measurements favor light primaries at these energies (see section 3), for this energy bin too an extragalactic origin of UHECR is favored by our data.

Regarding the search for small and intermediate scale correlation at high energies (above $\sim$ $10^{19} \mathrm{eV}$ ), no observation has been made so far. However, a few interesting indications of anisotropy have been found. First of all, the region around Centaurus A, the closest active galaxy, shows an 

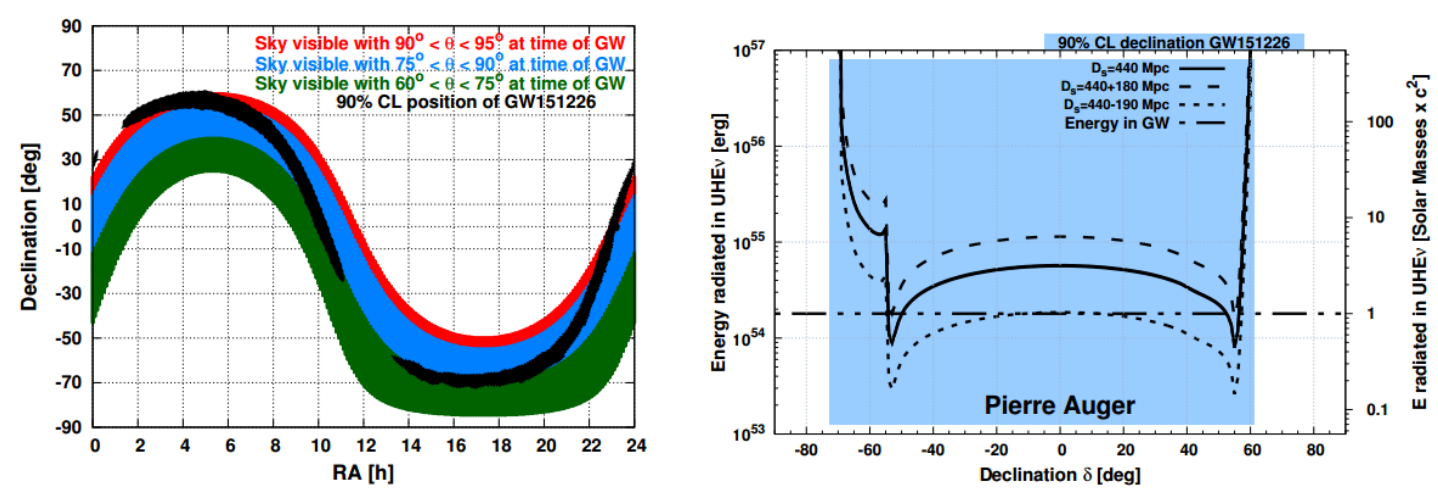

Figure 4: Left: Auger sky coverage at different zenith angles at the time of the detection of GW151226. The black contour region corresponds to the $90 \%$ C.L. region for the GW event location. Right: Constraints on the radiated energy in UHE neutrinos (per flavor) from the source of GW151226 as a function of declination. Energies above the solid line are excluded at the 90\% C.L. For more information see [13]

excess of UHECRs with energy higher than $37 \mathrm{EeV}$ observed up to August 2018. Inside a circle of radius $\psi=28^{\circ}$ centered on CenA, 203 events were observed while 141 were expected, and the local significance is $5.1 \sigma$. This significance has to be penalized because we performed a scan on the energy threshold and angular scale of the excess, leading to a one-sided post-trial significance of $3.9 \sigma$ [14]. This excess is steadily growing from earlier publications, such as [15], where only events up to March 2014 were considered.

An even more significant departure from isotropy was found when searching for correlation with catalogs of candidate sources, taking into account also their luminosity, under the assumption that the UHECR flux is proportional to the non-thermal electromagnetic flux. The search was performed through a maximum likelihood method that took into account the absorption due to the interaction of UHECRs during their propagation from each source.The likelihood analysis was performed on four catalogs: first, the 2MRS [18], taking out sources closer than $1 \mathrm{Mpc}$, as selected in [17], which trace the nearby matter. Secondly, the AGNs observed by the BAT camera on the Swift satellite [19], which include both radio loud and quiet AGNs. Then $\gamma-$ AGNs, selected from the 3FHL catalog [20] were used. Finally, the last catalog used is a sample of starburst galaxies selected based on their continuum emission at $1.4 \mathrm{GHz}$, used as a proxy for their UHECR flux. This selection has been updated by merging the data from [21] and [22] already reported in [17], with the addition of the Circinus Galaxy and sources selected with HEASARC Radio Master Catalog. ${ }^{2}$ The number of sources selected this way is 32 . The best-fit test statistics (TS) obtained for each catalog at each energy threshold are shown in figure 5, at left for starburst and $\gamma$-AGN and at right for Swift-BAT and 2MRS. The maximum likelihood-ratio is found with starburst galaxies for $E>38 \mathrm{EeV}$, with a TS of 29.5. At this energy threshold, the best-fit parameters are $f_{\text {aniso }}=11_{-4}^{+5} \%$ and $\theta=15_{-4}^{+5}{ }^{\circ}$, corresponding to a local p-value of $4 \times 10^{-7}$, which has then to be penalized for the scan on energy threshold, resulting in a post-trial significance of $4.5 \sigma$. A summary of the best-fit parameters obtained for all the four catalogs is reported in table 1.

\footnotetext{
${ }^{2}$ https://heasarc.nasa.gov/W3Browse/master-catalog/radio.html
} 

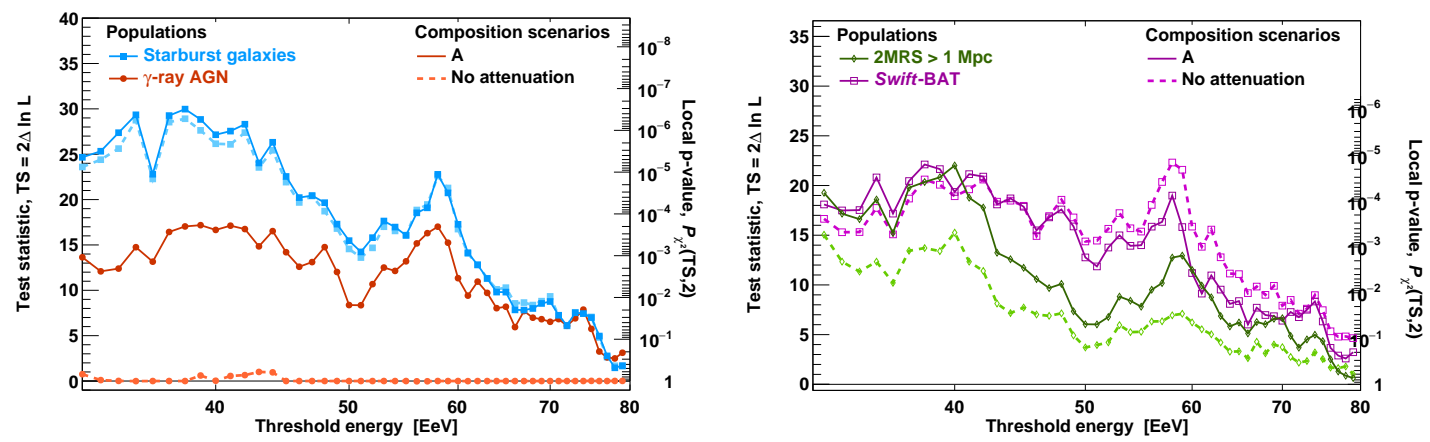

Figure 5: Maximum likelihood-ratio as a function of energy threshold for the models based on starburst galaxies and $\gamma$-AGN (left) and Swift-BAT and 2MRS (right). The dashed lines are the same obtained in the no-attenuation scenario.

\begin{tabular}{c||c|c|c|c|c|c} 
Catalog & $E_{\text {th }}$ & TS & Local p-value & post-trial & $f_{\text {aniso }}$ & $\theta$ \\
\hline Starburst & $38 \mathrm{EeV}$ & 29.5 & $4 \times 10^{-7}$ & $4.5 \sigma$ & $11_{-4}^{+5} \%$ & $15_{-4}^{+5 \circ}$ \\
$\gamma-$ AGN & $39 \mathrm{EeV}$ & 17.8 & $1 \times 10^{-4}$ & $3.1 \sigma$ & $6_{-3}^{+4} \%$ & $14_{-4}^{+6} \circ$ \\
Swift-BAT & $38 \mathrm{EeV}$ & 22.2 & $2 \times 10^{-5}$ & $3.7 \sigma$ & $8_{-3}^{+4} \%$ & $15_{-4}^{+6} \circ$ \\
2 MRS & $40 \mathrm{EeV}$ & 22.0 & $2 \times 10^{-5}$ & $3.7 \sigma$ & $19_{-7}^{+10} \%$ & $15_{-4}^{+7} \circ$ \\
\hline
\end{tabular}

Table 1: Values of the parameters that maximize the likelihood-ratio test against isotropy for the four different models as described in the text.

\section{AugerPrime: the Auger upgrade}

It is evident that the UHECR mass composition is a key parameter and that, at the highest energies, it cannot at present be well determined, in particular on an event-by-event basis. For this reason, the Collaboration is now working on a major upgrade of the detector, called AugerPrime [26]. A number of improvements are foreseen for this upgrade, the perhaps most important of them being the deployment of plastic scintillators of an approximate area of $\sim 4 \mathrm{~m}^{2}$ on top of each SD station (SSD). The two detectors will respond differently to the electromagnetic and muonic components of the EAS, and by comparison of the signal in each of them, a direct estimation of the muonic signal can be computed. A small portion of the array will benefit also from buried muon detectors that can calibrate and validate the measurements made with the SSD. Additionally, each SD station will be equipped with faster electronics and a new PMT, much smaller than the existing ones, to improve the dynamic range of each station. On the FD side, the uptime will be extended to partially moon-illuminated nights by decreasing the gain of the PMTs in the FD cameras. At present, the upgrade is being installed in the field, with SSDs already deployed on a large fraction of the SD stations.

\section{Conclusions and outlook}

This year the Pierre Auger Observatory celebrated its $20^{\text {th }}$ anniversary, and in these two decades, it has enormously improved our knowledge of ultra-high energy cosmic rays. In par- 
ticular, it has measured with high precision the flux of those particles, studied the characteristics of the air showers they induce and, within certain limits, measured their composition. Studying the large scale distribution of UHECRs, Auger was able to infer at which energy the transition between Galactic and extragalactic cosmic ray sources happens. Moreover, important results are being published regarding the arrival directions of the highest-energy events. Still, there is lots of work to be done: in particular, the astrophysical sources of these particles, the most energetic ones known in the universe, are still unknown. A better understanding of those open questions will be strongly supported by Auger upgrade, AugerPrime, that will allow for a better understanding of the composition at the highest energies.

\section{References}

[1] The Pierre Auger Collaboration, Nucl. Instrum. Meth. A 798 (2015) 172.

[2] The Pierre Auger Collaboration, Science 357 (2017) 1266-1270

[3] V. Verzi for The Pierre Auger Collaboration. Proceedings for the 36th International Cosmic Ray Conference Madison, WI, USA.. POS (ICRC2019) 450

[4] V. Verzi for The Pierre Auger Collaboration. Proceedings for the 33rd International Cosmic Ray Conference (ICRC 2013) arXiv:1307.5059

[5] E. Roulet for The Pierre Auger Collaboration, Proceedings for the 36th International Cosmic Ray Conference Madison, WI, USA.. POS (ICRC2019) 408

[6] V. Novotny for The Pierre Auger Collaboration, Proceedings for the 36th International Cosmic Ray Conference Madison, WI, USA.. POS ( ICRC2019) 374

[7] A. Coleman for The Pierre Auger Collaboration, Proceedings for the 36th International Cosmic Ray Conference Madison, WI, USA.. POS (ICRC2019) 225

[8] A. Yushkov for The Pierre Auger Collaboration, Proceedings for the 36th International Cosmic Ray Conference Madison, WI, USA.. POS (ICRC2019) 482

[9] J. Rautenberg for The Pierre Auger Collaboration, Proceedings for the 36th International Cosmic Ray Conference - Madison, WI, USA.. POS (ICRC2019) 398

[10] F. Pedreira for The Pierre Auger Collaboration, Proceedings for the 36th International Cosmic Ray Conference Madison, WI, USA.. POS (ICRC2019) 979

[11] The Pierre Auger Collaboration, Phys.Lett. B762 (2016) 288-295

[12] C. J. Todero Peixoto for The Pierre Auger Collaboration, Proceedings for the 36th International Cosmic Ray Conference - Madison, WI, USA.. POS (ICRC2019) 440

[13] The Pierre Auger Collaboration, Phys. Rev. D 94 (2016) 122007

[14] L. Caccianiga for The Pierre Auger Collaboration, Proceedings for the 36th International Cosmic Ray Conference - Madison, WI, USA.. POS (ICRC2019) 206

[15] The Pierre Auger Collaboration, Astrophys. J 804 (2015) 15

[16] U. Giaccari for The Pierre Auger Collaboration,Proceedings for the 35th International Cosmic Ray Conference. POS (ICRC2017) 483

[17] The Pierre Auger Collaboration, Astrophys. J. Lett. 853 (2018) L29

[18] J. P. Huchra, L. M. Macri, K. L. Masters, et al. Astrophys. J. Supp. 199 (2012) 26

[19] W. H. Baumgartner et al., Astrophys. J. Supp. 207 (2013) 19

[20] M. Ajello, et al. Astrophys. J. Supp. 232.2 (2017) 18

[21] M. Ackermann, M. Ajello,A. Allafort, et al. , Astrophys. J. 755 (2012) 164

[22] Becker, J. K., Biermann, P. L., Dreyer, J, Kneiske, T. M. 2009, ArXiv e-prints, arXiv:0901.1775 
[23] The Pierre Auger Collaboration, Astroparticle Physics 27 (2007) 244

[24] The Pierre Auger Collaboration, Astrophys. J. , 760 (2012) 148

[25] The Pierre Auger Collaboration, Phys. Rev. D 96 (2017) 122003

[26] The Pierre Auger Collaboration, arXiv:1604.03637

[27] The Pierre Auger Collaboration, PRL, 116 (2016) 241101

\section{DISCUSSION}

WOLFGANG KUNDT: How far do you feel can you observe at the highest CR energies?

LORENZO CACCIANIGA: The expected horizon depends on the composition. It is expected to be of the order of $\sim 100 \mathrm{Mpc}$ for protons and iron nuclei. For an intermediate composition, this value is expected to be lower. Exotic scenarios such as the presence of a Lorentz Invariance Violation may increase this horizon by a huge factor.

LUIGI COSTAMANTE: Given the poor knowledge of the hadronic interactions at such high energies, how much this affects the measure of $X_{\max }$ and $\sigma\left(X_{\max }\right)$, in particular its trend towards "heavier" composition. Could it be pure protons, and the flattening due to some unknown process/channel at these energies?

LORENZO CACCIANIGA: As far as we know we don't have reasons to believe that the dependence of $X_{\max }$ from energy can have abrupt slope changes, as we see in data. Using Occam's Razor, it is more prudent to suppose that the $X_{\max }$ evolution with energy is due to a change in composition, and at the highest energies this would mean a change towards heavier composition. 